\title{
Use of BAR score as predictor of short and long-term survival of liver transplantation patients
}

\author{
Chung-Mau Lo
}

Received: 25 September 2014 / Accepted: 8 October 2014/Published online: 31 October 2014

(C) Asian Pacific Association for the Study of the Liver 2014

In an attempt to reduce waiting time and waiting list mortality, decision making in liver transplantation in recent years has been largely dominated by two strategies: prioritization of the sickest patients for transplantation using the model for end-stage liver disease (MELD) score [1] and increased use of high-risk donor livers. The combination of these has, however, frequently created the difficult situation of a high-risk donor liver for a high-risk patient, leading to expected poor outcome and the possibility of a futile transplant. Furthermore, waiting list mortality must be balanced against survival outcome after transplantation to optimize utility and justice in organ allocation and resource utilization. Hence, different models have been developed to predict the outcome of transplantation. Models based either on donor factors alone (for example donor risk index, DRI) [2] or on recipient factors alone (for example MELD score) [1] are not predictive of survival after liver transplantation. Others that incorporated both donor and recipient factors have achieved better accuracy. The survival outcomes following liver transplantation (SOFT) score [3] is a complex list of 18 factors and the D-MELD [4] simply combines two simple dominant factors for donors and recipients. More recently, the balance of risk (BAR) score [5], which includes six variables (donor age, recipient age, recipient MELD score, retransplantation, pretransplant life support, and cold ischemic time) has been developed using the United Network for Organ Sharing (UNOS) database of the United States of America and validated in a European center.

C.-M. Lo $(\bowtie)$

Department of Surgery, Queen Mary Hospital, The University of Hong Kong, Pokfulam, Hong Kong, China

e-mail: chungmlo@hku.hk
In this issue of the journal, Campos Junior and colleagues attempt to validate the BAR score as a predictor of survival outcome for a cohort of 402 patients after liver transplantation in a single center in Brazil [6]. Although the BAR score with a cutoff of 11 has prognostic significance for 3 and 12-month survival, its predictive accuracy is low, as indicated by an area under the receiver operating curve (AUROC) of only 0.65 for this Brazilian population. In fact, multiple regression analysis for the prognostic factors of three and twelve-month survival does not identify any of the six variables incorporated in the BAR score as of prognostic significance.

Several differences between this study population and the UNOS database may account for the poor prognostic value of the BAR score for this cohort of patients. First, the recipients from Brazil were younger (median age 48.8 vs 54 years) and had a higher MELD score (median of $20 \mathrm{vs}$ 18). Second, the donors were also younger (median age of 35.6 vs 43 years) and had a higher DRI (median 1.68 vs 1.38). Third, cold ischemic time was much longer (median 10 vs $7 \mathrm{~h}$ ). Hence, there seems to be marked differences for at least four of six of the BAR score associated variables between the Brazilian cohort and the UNOS database [5]. Fourth, and more important, survival outcome for this Brazilian cohort was much worse, with three-month survival of $77 \%$ for those with a BAR score below 11 and $46 \%$ for those with a score greater than or equal to 11 . Although the authors did not report three-month survival for the entire cohort, the figure should be at or below $70 \%$ and was much lower than that from the UNOS database (93.2\%). Although the different donor and recipient characteristics, especially the higher MELD score and DRI, might have contributed to the inferior outcome, the possibility of a surgical or center factor cannot be excluded. In fact, the authors identify an operative factor, transfusion of 
more than six units of packed red cells, as an independent risk factor for survival at both 3 and 12 months.

Mortality on the waiting list for liver transplantation depends on a patient's disease severity and can be accurately predicted by use of the MELD score, which has been shown to be applicable to different patient populations. The outcome after a major operation such as liver transplantation, however, depends on many other factors and a predictive model is much more complex. For obvious reasons, both donor and recipient factors are important but it is also likely that many operative factors may contribute to the outcome. Current models such as the BAR score or SOFT score have included ischemic time alone as the only operative factor, because it is predictable and, to some extent, controllable before transplantation. Identification and inclusion of other operative variables in a model may improve its accuracy but will reduce its usefulness in clinical practice.

Putting aside the issues of model deficiency, another issue related to application of the BAR score is the best cutoff for the decision to transplant or not. What is the definition of a futile transplant and should there be an internationally acceptable standard? In the original study that proposed the BAR score [5], a threshold of the BAR score at 18 was postulated, beyond which survival deteriorated sharply; this was, hence, defined as a futile transplant. The Brazilian study, however, found a cut-off at a BAR score of 11 only, above which three-month survival was $46 \%$. If this is taken as the threshold for a futile transplant, many recipients will not be acceptable or will never be able to obtain a liver graft for transplantation in Brazil. For example, a MELD score $>35$ (BAR score of 14) will be regarded as a contraindication for transplantation. All candidates with a MELD score between 25 and 35 (BAR score of 10) will only be transplanted if they are aged 40 or less, have no previous transplant, and do not need life support pretransplant, and the liver graft will have to come from a perfect donor of age 40 or below and the coldischemic time should be $6 \mathrm{~h}$ or less. Even for patients with a MELD score between 15 and 25 (BAR score of 5), retransplantation (BAR score of 4) will not be acceptable if they are either older than 60 (BAR score of 3), on life support pretransplant (BAR score of 3 ), or the donor is older than 40 and the ischemic time is more than $6 \mathrm{~h}$ (BAR score of 2). Adopting the use of BAR score in this manner would bar many patients from the benefit of liver transplantation.

These results call for caution in adopting statistical models for decision making in patient care and policy making. A similar study based on two German cohorts [7] revealed similarly low predictive accuracy of the BAR score, as indicated by an AUROC of 0.66 , providing further support that the performance of the BAR score may be below the accepted threshold for potentially useful clinical prognostic models. The BAR score remains to be validated for larger patient populations and better models incorporating more variables may be necessary. Nonetheless, liver transplantation is a highly complex procedure. It may never be possible to find a model robust enough to be applicable across different centers and countries.

\section{References}

1. Wiesner RH, Edwards E, Freeman R, Harper A, Kim R, Kamath PS, et al. The United Network for Organ Sharing Liver Disease Score Committee. Model for end-stage liver disease (MELD) and allocation of donor livers. Gastroenterology. 2003;124:91-96

2. Feng S, Goodrich NP, Bragg-Gresham JL, Dykstra DM, Punch JD, DebRoy MA, et al. Characteristics associated with liver graft failure: the concept of a donor risk index. Am J Transplant. 2006;6:783-790

3. Rana A, Hardy MA, Halazun KJ, Woodland DC, Ratner LE, Samstein B, et al. Survival outcomes following liver transplantation (SOFT) score: a novel method to predict patient survival following liver transplantation. Am J Transplant. 2008;8: $2537-2546$

4. Halldorson JB, Bakthavatsalam R, Fix O, Reyes JD, Perkins JD. D-MELD, a simple predictor of post liver transplant mortality for optimization of donor/recipient matching. Am J Transplant. 2009;9:318-326

5. Dutkowski P, Oberkofler CE, Slankamenac K, Puhan MA, Schadde E, Müllhaupt B, et al. Are there better guidelines for allocation in liver transplantation? A novel score targeting justice and utility in the model for endstage liver disease era. Ann Surg. 2011;254:745-753

6. Campos Junior ID, Stucchi RSB, Udo EY, Boin IFSF. Application of the BAR score as a predictor of short- and long-term survival in liver transplantation patients. Hepatol Int. 2014. doi:10.1007/ s12072-014-9563-3

7. Schrem H, Platskis AL, Kaltenborn A, Koch A, Metz C, Barthold $\mathrm{M}$, et al. Value and limitations of the BAR-score for donor allocation in liver transplantation. Langenbecks Arch Surg. 2014. doi:10.1007/s00423-014-1247-x 